\title{
Sturm-Liouvilleov problem
}

\author{
Marija Čatipović, Saša Krešić-Jurić
}

\section{Sažetak}

Klasična Sturm-Liouvilleova jednadžba, nazvana po Jacquesu Sturmu i Josephu Liouvilleu, obična je diferencijalna jednadžba drugog reda oblika

$$
\frac{d}{d x}\left(p(x) \frac{d u}{d x}\right)+q(x) u=-\lambda w(x) u .
$$

Vrijednost $\lambda$ nije određena i pronalaženje te vrijednosti za koju postoje netrivijalna rješenja jednadžbe (1) i koja zadovoljavaju rubne uvjete dio je problema koji nazivamo Sturm-Liouvilleov problem. Pokazat ćemo da se proizvoljni linearni operator drugog reda može transformirati u SturmLiouvilleov operator tj. da je Sturm-Liouvilleov operator kanonski oblik diferencijalnog operatora drugog reda. Vlastite vrijednosti regularnog Sturm-Liouvilleovog problema su realne, prebrojive i tvore strogo rastući niz $\lambda_{1}<\lambda_{2}<\lambda_{3}<\ldots$ tako da vrijedi $\lim _{n \rightarrow \infty} \lambda_{n}=\infty$. Također, za svaku vlastitu vrijednost $\lambda_{n}$ postoji odgovarajuća vlastita funkcija $u_{n}(x)$ jedinstveno određena do na multiplikativnu konstantu koja ima točno $n$ nultočaka $\mathrm{u}$ intervalu $[a, b]$. Ovo je jedan od fundamentalnih rezultata za Sturm-Liouvilleove operatore.

Ključni pojmovi: Sturm-Liouvilleov problem, vlastite vrijednosti, vlastite funkcije, regularni

\section{Vrste Sturm-Liouvilleovog problema}

Promotrimo diferencijalnu jednadžbu oblika

$$
L u+\lambda w(x) u=0
$$


gdje je $L$ diferencijalni operator definiran sa

$$
L u=\frac{d}{d x}\left(p(x) \frac{d u}{d x}\right)+q(x) u .
$$

Diferencijalni operator $L$ nazivamo Sturm-Liouvilleov operator. Diferencijalne jednadžbe oblika (2) javljaju se kod rješavanja parcijalnih diferencijalnih jednadžbi matematičke fizike metodom separacije varijabli. Ako je $\hat{L}$ proizvoljni diferencijalni operator drugog reda

$$
\hat{L} u=a_{2}(x) \frac{d^{2} u}{d x^{2}}+a_{1}(x) \frac{d u}{d x}+a_{0}(x) u, \quad a_{2}(x) \neq 0, \forall x \in[a, b],
$$

tada se $\hat{L}$ može transformirati u Sturm-Liouvilleov operator množenjem sa funkcijom

$$
s(x)=\frac{1}{a_{2}(x)} \exp \left(\int \frac{a_{1}(x)}{a_{2}(x)} d x\right) .
$$

Doista, uočavamo da vrijedi

$$
(s(x) \hat{L}) u=\frac{d}{d x}\left(p(x) \frac{d u}{d x}\right)+q(x) u
$$

gdje je

$$
p(x)=\exp \left(\int \frac{a_{1}(x)}{a_{2}(x)} d x\right), \quad q(x)=\frac{a_{0}(x)}{a_{2}(x)} \exp \left(\int \frac{a_{1}(x)}{a_{2}(x)} d x\right) .
$$

Iz ovoga proizlazi da je Sturm-Liouvilleov operator kanonski oblik diferencijalnog operatora drugog reda.

Definicija 1. Regularni Sturm-Liouvilleov problem sastoji se od diferencijalne jednadžbe

$$
L u+\lambda w(x) u=0, \quad a \leq x \leq b,
$$

gdje je L operator oblika (3) $i$ rubnih uvjeta

$$
\begin{aligned}
a_{1} u(a)+a_{2} u^{\prime}(a) & =0, \\
b_{1} u(b)+b_{2} u^{\prime}(b) & =0 .
\end{aligned}
$$

gdje je $a_{1}^{2}+a_{2}^{2}>0 i b_{1}^{2}+b_{2}^{2}>0$. Funkcije $p, p^{\prime}$, q $i w$ su neprekidne na $[a, b]$ i $p(x)>0, w(x)>0 \forall x \in[a, b]$.

Kada je Sturm-Liouvilleov problem definiran na otvorenom ili poluotvorenom intervalu, ili ako $p(x)$ ili $w(x)$ isčezavaju u jednoj ili obje rubne točke intervala $[a, b]$, ili ako $p(x)$ ili $w(x)$ u rubnoj točki konvergiraju ka beskonačnosti, tada se on naziva singularni Sturm-Liouvilleov problem.

Također, Sturm-Liouvilleov problem je singularan ako je definiran na beskonačnom intervalu [3, 4, 5]. 
Definicija 2. U Sturm-Liouvilleovom problemu domena operatora $L$, koju označavamo sa $D(L)$, prostor je svih kompleksnih funkcija $u:[a, b] \rightarrow$ $\mathbb{C}$ za koje vrijedi $u^{\prime \prime} \in L^{2}([a, b])$. Tada imamo $L: D(L) \rightarrow L^{2}([a, b])$.

Za funkciju $u:[a, b] \rightarrow \mathbb{C}$ kažemo da je kvadratno integrabilna ako je $\int_{a}^{b}|u(x)|^{2} d x$ konačan. Prostor svih takvih funkcija označavamo sa $L^{2}([a, b])$ i zovemo Lebesgueovim prostorom kvadratno integrabilnih funkcija [1, 2.

Još jedan tip problema koji se često javlja u primjenama jest periodični Sturm-Liouvilleov problem. Sturm-Liouvilleova jednadžba

$$
L u+\lambda w(x) u=0, \quad a \leq x \leq b,
$$

gdje je $p(a)=p(b)$, zajedno s periodičnim rubnim uvjetima $u(a)=u(b)$, $u^{\prime}(a)=u^{\prime}(b)$ naziva se periodični Sturm-Liouvilleov problem.

\section{Vlastite vrijednosti i vlastite funkcije Sturm-Liouvilleovog problema}

Definicija 3. Funkcija u koja zadovoljava jednadžbu

$$
L u+\lambda w(x) u=0
$$

naziva se vlastita funkcija s pripadnom vlastitom vrijednošću $\lambda[6]$ ].

Vlastite vrijednosti $\lambda$ ovise o obliku operatora $L$ i o rubnim uvjetima koje zadovoljava funkcija $u$, kako ilustriraju sljedeći primjeri.

Primjer 4. Promotrimo diferencijalnu jednadžbu

$$
u^{\prime \prime}+\lambda u=0, \quad 0 \leq x \leq \pi
$$

s rubnim uvjetima $u(0)=u(\pi)=0$.

Uočavamo da je ovo regularni Sturm-Liouvilleov problem gdje je $p(x)=$ $w(x)=1, q(x)=0$. Promotrimo sve moguće slučajeve u ovisnosti o vrijednosti $\lambda$.

Ako je $\lambda<0$, tada je opće rješenje jednadžbe (7) dano sa

$$
u(x)=A e^{\sqrt{|\lambda|} x}+B e^{-\sqrt{|\lambda|} x} .
$$

Rubni uvjeti su zadovoljeni ako i samo ako je

$$
\begin{aligned}
& u(0)=A+B=0 \\
& u(\pi)=A e^{\sqrt{|\lambda|} \pi}+B e^{-\sqrt{|\lambda|} \pi}=0 .
\end{aligned}
$$


Kako je determinanta matrice sustava $8-(9)$

$$
\left|\begin{array}{cc}
1 & 1 \\
e^{\sqrt{|\lambda|} \pi} & e^{-\sqrt{|\lambda|} \pi}
\end{array}\right|=e^{-\sqrt{|\lambda|} \pi}-e^{\sqrt{|\lambda|} \pi} \neq 0,
$$

sustav (8)- 9 ima samo trivijalno rješenje $A=B=0$. Dakle, sustav nema netrivijalnih rješenja za $\lambda<0$ pa sustav nema vlastite vrijednosti za $\lambda<0$. Ako je $\lambda=0$, tada je opće rješenje jednadžbe (7) dano sa

$$
u(x)=A+B x .
$$

Iz rubnih uvjeta

$$
\begin{aligned}
& u(0)=A=0, \\
& u(\pi)=A+B \pi=0,
\end{aligned}
$$

slijedi da je $A=B=0$. Dakle, $\lambda=0$ također nije vlastita vrijednost sustava. Neka je sada $\lambda>0$. Tada je opće rješenje jednadžbe (7) dano sa

$$
u(x)=A \cos (\sqrt{\lambda} x)+B \sin (\sqrt{\lambda} x)
$$

pa rubni uvjeti impliciraju

$$
\begin{aligned}
& u(0)=A \cdot 1+0=A=0, \\
& u(\pi)=A \cos (\sqrt{\lambda} \pi)+B \sin (\sqrt{\lambda} \pi)=0 .
\end{aligned}
$$

Ovaj sustav ima netrivijalna rješenja ako je

$$
\sin (\sqrt{\lambda} \pi)=0
$$

odnosno $\sqrt{\lambda} \pi=n \pi$, za $n=1,2,3, \ldots$ Dakle, vlastite su vrijednosti dane sa

$$
\lambda_{n}=n^{2}, \quad n=1,2,3, \ldots
$$

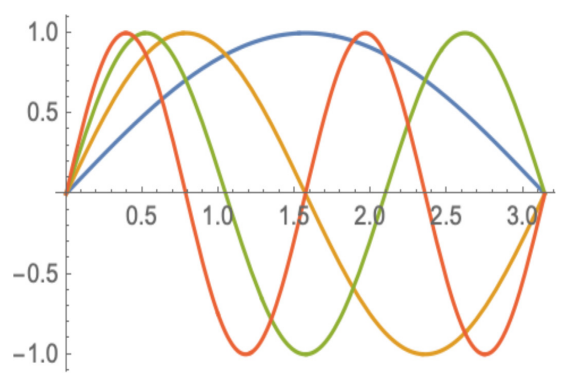

Slika 1. Vlastite funkcije $u_{n}(x)=\sin (n x), n=1,2,3,4$. 
a pripadne vlastite funkcije (do na multiplikativnu konstantu) su

$$
u_{n}(x)=\sin (n x) .
$$

Uočimo da $\lambda_{n} \rightarrow \infty$ kada $n \rightarrow \infty$; što je tipično za vlastite vrijednosti Sturm-Liouvilleovog problema.

Primjer 5. Promotrimo Eulerovu jednadžbu

$$
x^{2} u^{\prime \prime}+x u^{\prime}+\lambda u=0, \quad 1 \leq x \leq e,
$$

s rubnim uvjetima

$$
u(1)=0, \quad u(e)=0 .
$$

Eulerova jednadžba se može napisati u Sturm-Liouvilleovom obliku

$$
\frac{d}{d x}\left(x \frac{d u}{d x}\right)+\frac{1}{x} \lambda u=0
$$

gdje je $w(x)=\frac{1}{x}, p(x)=x$, a $q(x)=0$. Rješenje ove jednadžbe dano je sa

$$
u(x)=c_{1} x^{i \sqrt{\lambda}}+c_{2} x^{-i \sqrt{\lambda}},
$$

gdje je $x^{i a}=e^{i a \ln x}=\cos (a \ln x)+i \sin (a \ln x)$. Stoga rješenje $u(x)$ prelazi u oblik

$$
u(x)=A \cos (\sqrt{\lambda} \ln x)+B \sin (\sqrt{\lambda} \ln x),
$$

gdje su $A$ i $B$ konstante vezane uz $c_{1}$ i $c_{2}$. Rubni uvjeti impliciraju sljedeće:

$$
\begin{aligned}
& u(1)=A \cos (\sqrt{\lambda} \ln 1)+B \sin (\sqrt{\lambda} \ln 1)=A=0, \\
& u(e)=A \cos (\sqrt{\lambda} \ln e)+B \sin (\sqrt{\lambda} \ln e)=A \cos (\sqrt{\lambda})+B \sin (\sqrt{\lambda})=0 .
\end{aligned}
$$

Ovaj sustav ima netrivijalna rješenja ako je $\sin (\sqrt{\lambda})=0, B \neq 0$, odnosno $\sqrt{\lambda}=n \pi$, za $n=1,2,3, \ldots$ Dakle, vlastite vrijednosti dane su sa

$$
\lambda_{n}=n^{2} \pi^{2}, \quad n=1,2,3, \ldots,
$$

a pripadne vlastite funkcije su

$$
u_{n}(x)=\sin (n \pi \ln x), \quad n=1,2,3, \ldots
$$

Primjer 6. Odredimo vlastite vrijednosti i vlastite funkcije periodičnog Sturm-Liouvilleovog problema

$$
u^{\prime \prime}+\lambda u=0, \quad-\pi \leq x \leq \pi
$$

s periodičnim rubnim uvjetima

$$
u(-\pi)=u(\pi), \quad u^{\prime}(-\pi)=u^{\prime}(\pi) .
$$


Uočavamo da je $p(x)=1$, pa je uvjet $p(-\pi)=p(\pi)$ ispunjen. Kada je $\lambda>0$, opće rješenje Sturm-Liouvilleove jednadžbe dano je sa

$$
u(x)=A \cos (\sqrt{\lambda} x)+B \sin (\sqrt{\lambda} x) .
$$

Iz periodičnog rubnog uvjeta $u(-\pi)=u(\pi)$ dobivamo sljedeću jednakost

$$
2 B \sin (\sqrt{\lambda} \pi)=0 .
$$

Budući da je

$$
u^{\prime}(x)=-A \sqrt{\lambda} \sin (\sqrt{\lambda} x)+B \sqrt{\lambda} \cos (\sqrt{\lambda} x),
$$

uvjet $u^{\prime}(-\pi)=u^{\prime}(\pi)$ implicira

$$
2 A \sqrt{\lambda} \sin (\sqrt{\lambda} \pi)=0 .
$$

Dakle, ako vrijedi

$$
\sin (\sqrt{\lambda} \pi)=0,
$$

tada netrivijalna rješenja postoje, a vlastite vrijednosti dane su sa

$$
\lambda_{n}=n^{2}, \quad n=1,2,3, \ldots .
$$

Kako je jednadžba $\sin (\sqrt{\lambda} \pi)=0$ zadovoljena za proizvoljne $A$ i $B$, dobivamo dvije linearno nezavisne vlastite funkcije

$$
u_{n}(x)=\cos (n x) \text { i } u_{n}(x)=\sin (n x)
$$

koje odgovaraju istoj vlastitoj vrijednosti $\lambda_{n}=n^{2}$ za svaki $n=1,2,3, \ldots$ Slično kao u primjeru 4 uočavamo da za $\lambda<0$ rješenje Sturm-Liouvilleove jednadžbe ne zadovoljava periodične rubne uvjete. Ako je $\lambda=0$, tada rješenje jednadžbe

$$
u(x)=A+B x
$$

zadovoljava rubne uvjete ako je

$$
2 B \pi=0,
$$

odnosno $B=0$. Stoga je $\lambda=0$ vlastita vrijednost sa pripadnim rješenjem $u_{0}(x)=1$. Dakle, vlastite vrijednosti ovog problema su

$$
\lambda_{n}=n^{2}, \quad n=0,1,2, \ldots
$$

a pripadne vlastite funkcije su dane sa

$$
1, \cos (n x), \sin (n x), \quad n=1,2,3, \ldots
$$




\section{Svojstva vlastitih vrijednosti i vlastitih funkcija Sturm-Liouvilleovog problema}

Promotrimo sada neka važna svojstva Sturm-Liouvilleovog problema.

Teorem 7 (Lagrangeov identitet). Neka su u $i v$ funkcije $u$ domeni Sturm-Liouvilleovog operatora L. Tada vrijedi [3]

$$
u L v-v L u=\frac{d}{d x}\left(p\left(u v^{\prime}-v u^{\prime}\right)\right) .
$$

Dokaz. Koristeći definiciju (3) Sturm-Liouvilleovog operatora imamo:

$$
\begin{aligned}
u L v-v L u & =u \frac{d}{d x}\left(p \frac{d v}{d x}\right)+q u v-v \frac{d}{d x}\left(p \frac{d u}{d x}\right)-q u v \\
& =u \frac{d}{d x}\left(p \frac{d v}{d x}\right)-v \frac{d}{d x}\left(p \frac{d u}{d x}\right) \\
& =p^{\prime}\left(u v^{\prime}-v u^{\prime}\right)+p\left(u v^{\prime \prime}-v u^{\prime \prime}\right) \\
& =\frac{d}{d x}\left(p\left(u v^{\prime}-v u^{\prime}\right)\right) .
\end{aligned}
$$

Teorem 8 (Abelova formula). Ako su u $i v$ dva rješenja jednadžbe

$$
L u+\lambda w(x) u=0, \quad a \leq x \leq b,
$$

tada je $p(x) W(x ; u, v)$ konstanta, gdje je

$$
W(x ; u, v)=\left|\begin{array}{cc}
u & v \\
u^{\prime} & v^{\prime}
\end{array}\right|
$$

Wronskijan funkcija u i $v$ [3].

Dokaz. Prema pretpostavci $u$ i $v$ su rješenja jednadžbe $L u+\lambda w(x) u=0$, pa imamo

$$
\begin{aligned}
\frac{d}{d x}\left(p u^{\prime}\right)+(q+\lambda w(x)) u & =0 \\
\frac{d}{d x}\left(p v^{\prime}\right)+(q+\lambda w(x)) v & =0 .
\end{aligned}
$$

Množeći prvu jednadžbu s $v$, a drugu s $u$, oduzimanjem dobivamo

$$
v \frac{d}{d x}\left(p u^{\prime}\right)-u \frac{d}{d x}\left(p v^{\prime}\right)=0 .
$$

Parcijalnom integracijom jednakosti 10 imamo 


$$
\begin{aligned}
\int_{a}^{x} v \frac{d}{d t} & \left(p u^{\prime}\right) d t-\int_{a}^{x} u \frac{d}{d t}\left(p v^{\prime}\right) d t \\
& =\left.v(t) p(t) u^{\prime}(t)\right|_{a} ^{x}-\int_{a}^{x} p v^{\prime} u^{\prime} d t-\left.u(t) p(t) v^{\prime}(t)\right|_{a} ^{x}+\int_{a}^{x} p u^{\prime} v^{\prime} d t \\
& =p(x)\left(u^{\prime}(x) v(x)-u(x) v^{\prime}(x)\right)-p(a)\left(u^{\prime}(a) v(a)-u(a) v^{\prime}(a)\right) \\
& =p(x) W(x ; u, v)-p(a)\left(u^{\prime}(a) v(a)-u(a) v^{\prime}(a)\right)=0
\end{aligned}
$$

Iz toga slijedi da je $p(x) W(x ; u, v)$ konstanta.

Vlastitoj vrijednosti Sturm-Liouvilleovog problema može pripadati jedna ili više vlastitih funkcija. U primjeru 6 vlastita vrijednost $\lambda_{0}=0$ ima jednu vlastitu funkciju $u_{0}(x)=1$, dok svakoj vlastitoj vrijednosti $\lambda_{n}=n^{2}, n \geq 1$, pripadaju dvije vlastite funkcije $u_{n}(x)=\cos (n x) \mathrm{i}$ $u_{n}(x)=\sin (n x)$.

Definicija 9. Maksimalni broj linearno nezavisnih funkcija koje pripadaju istoj vlastitoj vrijednosti naziva se multiplicitet vlastite vrijednosti [1].

Teorem 10. Vlastite funkcije regularnog Sturm-Liouvilleovog problema jedinstvene su do na multiplikativnu konstantu [3].

Dokaz. Neka su $u$ i $v$ rješenja regularnog Sturm-Liouvilleovog problema

$$
L u+\lambda w(x) u=0, \quad a \leq x \leq b .
$$

Rubni uvjeti za funkcije $u$ i $v$ u točki $x=a$ daju

$$
\begin{aligned}
& a_{1} u(a)+a_{2} u^{\prime}(a)=0, \\
& a_{1} v(a)+a_{2} v^{\prime}(a)=0 .
\end{aligned}
$$

Barem jedan od koeficijenata $a_{1}$ i $a_{2}$ različit je od nule jer je po pretpostavci $a_{1}^{2}+a_{2}^{2}>0$, a to implicira da sustav (11) 12 ima netrivijalna rješenja za $a_{1}$ i $a_{2}$. Uočavamo da tada vrijedi

$$
W(a ; u, v)=\left|\begin{array}{cc}
u(a) & v(a) \\
u^{\prime}(a) & v^{\prime}(a)
\end{array}\right|=0 .
$$

Prema Abelovoj formuli je

$$
p(x) W(x ; u, v)=c, \quad a \leq x \leq b
$$

za neki $c \in \mathbb{R}$. Kada je $x=a$ imamo

$$
p(a) W(a ; u, v)=c,
$$


pa jednakost (13) implicira da je $c=0$ jer je $p(a)>0$. S obzirom da je Sturm-Liouvilleov problem regularan pa vrijedi $p(x)>0$ za svaki $x \in[a, b]$, iz jednakosti (14) slijedi

$$
W(x ; u, v)=0
$$

za svaki $x \in[a, b]$. Ovo implicira da su funkcije $u$ i $v$ linearno zavisne, tj. da vrijedi $u=\alpha v$ za neki $\alpha \in \mathbb{C}$.

Iz prethodnog teorema slijedi da vlastite vrijednosti regularnog SturmLiouvilleovog problema imaju multiplicitet jedan [3].

Ova tvrdnja je vidljiva u primjerima 4 i 5 . Ona ne vrijedi za periodični Sturm-Liouvilleov problem što se vidi iz primjera 6 .

Definicija 11. Skalarni umnožak na prostoru $L^{2}([a, b])$ s težinskom funkcijom $w, w(x)>0$ za sve $x \in[a, b]$ definiran je sa [1]

$$
\langle f, g\rangle_{w}=\int_{a}^{b} f(x) \overline{g(x)} w(x) d x .
$$

Ako je $w(x)=1$, za svaki $x \in[a, b]$, tada pišemo

$$
\langle f, g\rangle=\int_{a}^{b} f(x) \overline{g(x)} d x .
$$

Teorem 12. Neka su $u$ i $v$ funkcije u domeni Sturm-Liouvilleovog operatora $L$ koje zadovoljavaju rubne uvjete

$$
\begin{array}{ll}
a_{1} u(a)+a_{2} u^{\prime}(a)=0, & b_{1} u(b)+b_{2} u^{\prime}(b)=0, \\
a_{1} v(a)+a_{2} v^{\prime}(a)=0, & b_{1} v(b)+b_{2} v^{\prime}(b)=0,
\end{array}
$$

gdje je $a_{1}^{2}+a_{2}^{2}>0, b_{1}^{2}+b_{2}^{2}>0$. Tada je [3]

$$
\langle L u, v\rangle=\langle u, L v\rangle .
$$

Dokaz. Ako funkcija $v$ zadovoljava rubni uvjet (17), tada konjugirana funkcija $\bar{v}$ zadovoljava uvjete

$$
\begin{aligned}
a_{1} \bar{v}(a)+a_{2} \bar{v}^{\prime}(a) & =0, \\
b_{1} \bar{v}(b)+b_{2} \bar{v}^{\prime}(b) & =0,
\end{aligned}
$$

jer su $a_{i}$ i $b_{i}$ realni koeficijenti za $i=1,2$. Integracijom Lagrangeovog identiteta za funkcije $u$ i $\bar{v}$ dobivamo

$$
\begin{aligned}
\int_{a}^{b}(u L \bar{v}-\bar{v} L u) d x=\left.p(x)\left(u(x) \bar{v}^{\prime}(x)-\bar{v}(x) u^{\prime}(x)\right)\right|_{x=a} ^{x=b} \\
=p(b)\left(u(b) \bar{v}^{\prime}(b)-\bar{v}(b) u^{\prime}(b)\right)-p(a)\left(u(a) \bar{v}^{\prime}(a)-\bar{v}(a) u^{\prime}(a)\right)
\end{aligned}
$$


Prema uvjetima (16) i 18 funkcije $u$ i $\bar{v}$ zadovoljavaju

$$
\begin{aligned}
& a_{1} u(a)+a_{2} u^{\prime}(a)=0, \\
& a_{1} \bar{v}(a)+a_{2} \bar{v}^{\prime}(a)=0 .
\end{aligned}
$$

Sustav 20 -21 ima netrivijalna rješenja za $a_{1}$ i $a_{2}$ jer je barem jedan od koeficijenata $a_{1}$ i $a_{2}$ različit od nule, što implicira da je determinanta matrice sustava $20-21$ jednaka

$$
W(a ; u, \bar{v})=\left|\begin{array}{cc}
u(a) & \bar{v}(a) \\
u^{\prime}(a) & \bar{v}^{\prime}(a)
\end{array}\right|=0 .
$$

Slično se pokazuje $W(b ; u, \bar{v})=0$. Stoga slijedi

$$
\int_{a}^{b}(u L \bar{v}-\bar{v} L u) d x=0 .
$$

Koeficijenti u operatoru $L$ realne su funkcije pa vrijedi $L \bar{v}=\overline{L v}$, stoga jednakost (22) možemo pisati u obliku

$$
\int_{a}^{b} u \overline{L v} d x=\int_{a}^{b}(L u) \bar{v} d x
$$

odnosno

$$
\langle u, L v\rangle=\langle L u, v\rangle
$$

Iz navedenog slijedi da je Sturm-Liouvilleov operator $L$ hermitski operator jer vrijedi

$$
\langle u, L v\rangle=\langle L u, v\rangle, \quad \forall u, v \in D(L) .
$$

Teorem 13. Vlastite vrijednosti regularnog Sturm-Liouvilleovog problema su realne [3].

Dokaz. Neka je $\lambda$ vlastita vrijednost Sturm-Liouvilleovog problema i neka je $u$ pripadna vlastita funkcija. Tada je $u \neq 0$ i

$$
L u=-\lambda w(x) u, \quad a \leq x \leq b .
$$

Kako je operator Sturm-Liouvilleovog problema hermitski imamo

$$
\langle L u, u\rangle-\langle u, L u\rangle=0
$$

odnosno

$$
\langle-\lambda w u, u\rangle-\langle u,-\lambda w u\rangle=0 .
$$


Odavde slijedi

$$
\lambda\langle w u, u\rangle-\bar{\lambda}\langle u, w u\rangle=(\lambda-\bar{\lambda}) \int_{a}^{b}|u(x)|^{2} w(x) d x=0 .
$$

Kako je

$$
\int_{a}^{b}|u(x)|^{2} w(x) d x>0
$$

jer je $w(x)>0$ za sve $x \in[a, b]$, jednakost 23) implicira $\lambda-\bar{\lambda}=0$, odnosno $\lambda \in \mathbb{R}$.

Iz navedenih se primjera vidi da vlastite vrijednosti $\lambda_{n}$ Sturm-Liouvilleovog problema tvore rastući niz i $\lim _{n \rightarrow \infty} \lambda_{n}=\infty$. Ovo zapažanje vrijedi općenito i dano je sljedećim teoremom.

Teorem 14. Regularni Sturm-Liouvilleov problem ima beskonačan niz realnih vlastitih vrijednosti

$$
\lambda_{1}<\lambda_{2}<\lambda_{3}<\ldots
$$

takav da vrijedi

$$
\lim _{n \rightarrow \infty} \lambda_{n}=\infty
$$

$Z$ a svaku vrijednost $\lambda_{n}$ odgovarajuća vlastita funkcija $u_{n}(x)$ jedinstveno je određena do na konstantu i ima točno $n$ nultočaka u intervalu $[a, b]$ [3].

Umjesto dokaza, ilustrirajmo ovaj teorem sljedećim primjerom.

Primjer 15. Promotrimo Sturm-Liouvilleov problem

$$
\begin{gathered}
u^{\prime \prime}+\lambda u=0, \quad 0 \leq x \leq 1, \\
u(0)=0, u(1)+h u^{\prime}(1)=0, \text { gdje je } h>0 .
\end{gathered}
$$

Uočavamo da je $p(x)=1, q(x)=0, w(x)=1$. Rješenje ovog problema dano je sa

$$
u(x)=A \cos (\sqrt{\lambda} x)+B \sin (\sqrt{\lambda} x) .
$$

Rubni uvjet $u(0)=0$ daje $A=0$ pa imamo

$$
u(x)=B \sin (\sqrt{\lambda} x) .
$$

Iz uvjeta $u(1)+h u^{\prime}(1)=0$ slijedi

$$
B(\sin \sqrt{\lambda}+h \sqrt{\lambda} \cos \sqrt{\lambda})=0 .
$$


Kako tražimo netrivijalna rješenja $(B \neq 0)$, iz prethodne jednadžbe dobivamo

$$
\tan \sqrt{\lambda}=-h \sqrt{\lambda} .
$$

Ako definiramo $\alpha=\sqrt{\lambda}$, tada je

$$
\tan \alpha=-h \alpha .
$$

Ovo je transcendentalna jednadžba čija se rješenja mogu prikazati grafički pomoću presjeka grafova funkcija $\xi=\tan \alpha$ i $\xi=-h \alpha$, kao na slici 2 , Uočavamo da postoji beskonačno mnogo rješenja $\alpha_{n}$ za $n=1,2,3, \ldots$ Svako rješenje $\alpha_{n}$ daje odgovarajuću vlastitu vrijednost

$$
\lambda_{n}=\alpha_{n}^{2}, \quad n=1,2,3, \ldots
$$

Prema tome, postoji uređeni niz vlastitih vrijednosti

$$
\lambda_{1}<\lambda_{2}<\lambda_{3}<\ldots
$$

takav da vrijedi

$$
\lim _{n \rightarrow \infty} \lambda_{n}=\infty .
$$

Pripadna vlastita funkcija dana je sa

$$
u_{n}(x)=\sin \left(\sqrt{\lambda_{n}} x\right) .
$$

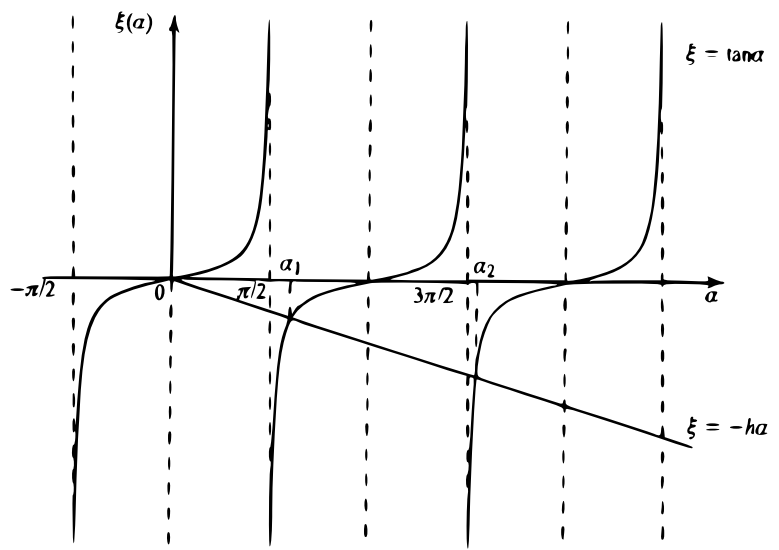

Slika 2. Presjeci grafova funkcija $\xi=\tan \alpha$ i $\xi=-h \alpha$.

Teorem 16. Vlastite funkcije koje pripadaju različitim vlastitim vrijednostima regularnog Sturm-Liouvilleovog operatora su ortogonalne u odnosu na skalarni umnožak $\langle,\rangle_{w}$ s težinskom funkcijom $w$ [4]. 
Dokaz. Neka su $u$ i $v$ vlastite funkcije regularnog Sturm-Liouvilleovog operatora $L$ koje pripadaju vlastitim vrijednostima $\lambda_{1} \neq \lambda_{2}$. Tada je

$$
\begin{aligned}
& L u=-\lambda_{1} w u, \\
& L v=-\lambda_{2} w v,
\end{aligned}
$$

Također vrijedi

$$
\langle L u, v\rangle=\langle u, L v\rangle
$$

jer je $L$ hermitski operator. Supstitucijom jednakosti (25) i (26) u jednakost (27) dobivamo

$$
\left\langle\lambda_{1} w u, v\right\rangle=\left\langle u, \lambda_{2} w v\right\rangle,
$$

odnosno, uzimajući u obzir da je $w$ realna funkcija i $\lambda_{1}, \lambda_{2} \in \mathbb{R}$, imamo

$$
\lambda_{1} \int_{a}^{b} w(x) u(x) \overline{v(x)} d x=\lambda_{2} \int_{a}^{b} u(x) w(x) \overline{v(x)} d x .
$$

Iz toga slijedi

$$
\left(\lambda_{1}-\lambda_{2}\right) \int_{a}^{b} u(x) \overline{v(x)} w(x) d x=0 .
$$

Kako je $\lambda_{1} \neq \lambda_{2}$, zaključujemo da je

$$
\langle u, v\rangle_{w}=\int_{a}^{b} u(x) \overline{v(x)} w(x) d x=0 .
$$

Dakle, $u$ i $v$ su ortogonalne s obzirom na skalarni umnožak (28) s težinskom funkcijom $w$.

\section{Primjer 17. Legendreova jednadžba}

$$
\frac{d}{d x}\left(\left(1-x^{2}\right) \frac{d u}{d x}\right)+\lambda u=0, \quad-1 \leq x \leq 1,
$$

s uvjetima da u i $u^{\prime}$ imaju konačan limes kada $x \rightarrow \pm 1$ singularni je Sturm-Liouvilleov problem.

$\mathrm{U}$ ovom problemu je $p(x)=1-x^{2}, q(x)=0, w(x)=1$ pri čemu $p(x)$ isčezava za $x= \pm 1$. Legendreove funkcije (Legendreovi polinomi)

$$
P_{n}(x)=\frac{1}{2^{n} n !} \frac{d^{n}}{d x^{n}}\left(\left(x^{2}-1\right)^{n}\right), \quad n=0,1,2, \ldots
$$

su vlastite funkcije, a $\lambda_{n}=n(n+1)$ su vlastite vrijednosti ovog SturmLiouvilleovog problema. Uočavamo da vlastitih vrijednosti ima beskonačno mnogo i da vrijedi $\lim _{n \rightarrow \infty} \lambda_{n}=\infty$. Također, vlastite funkcije $P_{n}(x)$ su ortogonalne u odnosu na skalarni produkt (15). 


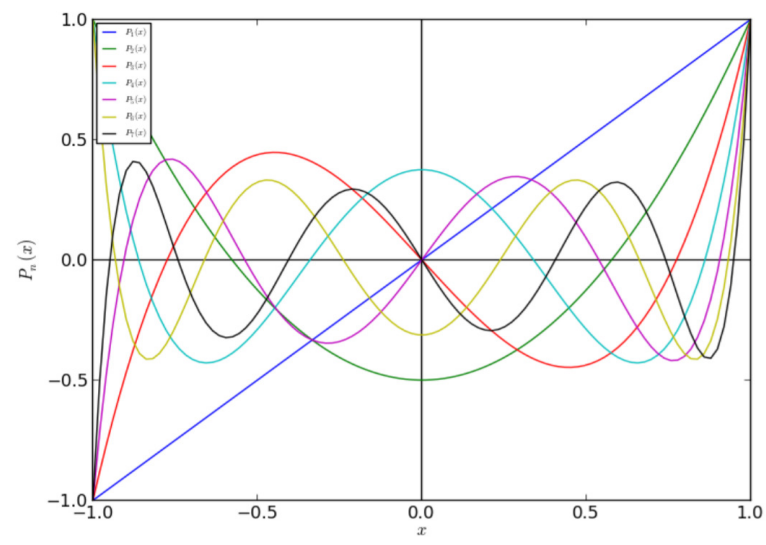

Slika 3. Legendreovi polinomi [7].

\section{Literatura}

[1] L. Debnath, P. Mikusinski, Introduction to Hilbert Spaces with Applications, drugo izdanje, Academic Press, San Diego, 1999.

[2] A. N. Kolomogorov, S. V. Fomin, Introductiory Real Analysis, drugo izdanje, Dover Publications, New York, 1970.

[3] T. Myint-U, L. Debnath, Linear Partial Differential Equations, četvrto izdanje, Boston, 2007

[4] Y. Pinchover, J. Rubinstein, An Introduction to Partial Differential Equations, Cambridge University Press, Cambrige, 2007.

[5] Web link, http://www.math.iitb.ac.in/ siva/ma41707/ode7. pdf Datum zadnjeg pristupa: 25.09.2020.

[6] Web link, http://www.macs.hw.ac.uk/ robertw/F13YT2/chap6. pdf, Datum zadnjeg pristupa: 20.09.2020.

[7] Web link, http://mathworld.wolfram.com/LegendrePolynomial. html, Datum zadnjeg pristupa: 25.09.2020.

Marija Čatipović

Sveučilište u Splitu, Fakultet elektrotehnike, strojarstva i brodogradnje, Ruđera Boškovića 32, 21000 Split, Hrvatska

E-mail adresa: mcatipov@fesb.hr 
Saša Krešić-Jurić

Sveučilište u Splitu, Prirodoslovno matematički fakultet, Ruđera Boškovića 33, 21000 Split, Hrvatska

E-mail adresa: skresic@pmfst.hr 